\title{
Phase diagram of the hard-core Bose-Hubbard model on a checkerboard superlattice
}

\author{
Itay Hen, ${ }^{1, *}$ M. Iskin, ${ }^{2, \dagger}$ and Marcos Rigol ${ }^{1, \dagger}$ \\ ${ }^{1}$ Department of Physics, Georgetown University, Washington, DC 20057, USA \\ ${ }^{2}$ Department of Physics, Koç University, Rumelifeneri Yolu, 34450 Sariyer, Istanbul, Turkey \\ (Received 4 November 2009; published 9 February 2010; corrected 26 February 2010)
}

\begin{abstract}
We obtain the complete phase diagram of the hard-core Bose-Hubbard model in the presence of a period-two superlattice in two and three dimensions. First we acquire the phase boundaries between the superfluid phase and the "trivial" insulating phases of the model (the completely-empty and completely-filled lattices) analytically. Next, the boundary between the superfluid phase and the half-filled Mott-insulating phase is obtained numerically, using the stochastic series expansion algorithm followed by finite-size scaling. We also compare our numerical results against the predictions of several approximation schemes, including two mean-field approaches and a fourth-order strong-coupling expansion, where we show that the latter method in particular is successful in producing an accurate picture of the phase diagram. Finally, we examine the extent to which several approximation schemes, such as the random phase approximation and the strong-coupling expansion, give an accurate description of the momentum distribution of the bosons inside the insulating phases.
\end{abstract}

DOI: 10.1103/PhysRevB.81.064503

PACS number(s): 67.85.-d, 64.70.Tg, 03.75.Lm, 02.70.Ss

\section{INTRODUCTION}

One of the most remarkable achievements in the field of ultracold Bose gases in recent years has been the observation of a superfluid-to-Mott-insulator transition in optical lattices. ${ }^{1}$ By playing with the intensity of the different laser beams involved in the setup, experimentalists have been able to study this transition in effective one- ${ }^{2}$ two-,${ }^{3}$ and three-dimensional ${ }^{1}$ geometries. This extraordinary accomplishment was achieved with gases of bosonic atoms confined in optical and magnetic traps. Using the strength of the optical lattice as a control parameter, these gases were reversibly tuned from a Bose-Einstein condensate to a Mott insulator (a state composed of localized atoms). ${ }^{4}$

It is generally accepted that this quantum phase transition can be studied using the Bose-Hubbard model, where the transition is found to be from a compressible superfluid phase to an incompressible Mott-insulating one (SF-MI). ${ }^{5}$ Over the years, much theoretical work has been devoted to determining the phase diagram of the model in various dimensions, using many different approaches. ${ }^{5-12}$ However, a direct comparison between theoretical results and experimental ones ${ }^{1-3}$ still remains obscured by issues such as the spatial inhomogeneity, ${ }^{13-15}$ finite-temperature effects, ${ }^{16,17}$ and the limited set of experimental tools available to probe the nearly isolated ultracold atomic systems.

In a recent paper, Aizenman et al..$^{18}$ argued that the phases of the Bose-Hubbard model can be studied equally well by examining a slightly different variant of it, namely, the BoseHubbard model in the limit of infinite onsite repulsion (i.e., the case of hard-core bosons), in the presence of an alternating (checkerboard) onsite chemical potential (a superlattice with period two). The advantage of studying the latter model lies in the fact that it exhibits all the salient properties of the Bose-Hubbard model while also being more amenable to analytical treatment. Specifically, Aizenman et al. rigorously proved the existence of SF and MI phases in the half-filled three-dimensional case (although they did not show that there is no intermediate phase between the two). In Ref. 19, two of us (Hen and Rigol) studied that very same model for the case of zero chemical potential both in two and three dimensions, using quantum Monte Carlo simulations and analytical approximation approaches. We showed that the SF-MI phase transition is a direct transition and we determined its critical value.

The hard-core Bose-Hubbard model with a superlattice has yet another attractive feature that the general BoseHubbard model lacks: it is exactly solvable in one dimension. This is due to the existence of a mapping of the hardcore bosons to noninteracting fermions. This in turn enables the evaluation of correlation functions of interest by exact means. ${ }^{20-22}$

In this paper, we study the complete phase diagram of hard-core bosons in the presence of a superlattice in two and three dimensions, and with arbitrary chemical potential. We determine the phase boundaries separating the compressible SF phase of the model from the various insulating phases. First we acquire the phase boundaries between the SF phase and the "trivial" insulating phases (the completely-empty and completely-filled lattices) analytically. Then we perform high-precision numerical simulations using the stochastic series expansion (SSE) algorithm ${ }^{23,24}$ in order to find the phase boundary of the transition between the SF and the half-filled MI. This is done by calculating the free energy $\Omega$, the density of bosons in the zero-momentum mode $\rho_{0}$, and the superfluid density $\rho_{s}$. The latter two quantities drop to zero upon entering the insulating regime from the SF phase.

Once the complete phase diagram is obtained, we proceed to examine the model analytically by employing two meanfield-type approximations and a strong-coupling perturbation scheme (up to fourth order in the hopping parameter) in order to determine the extent to which analytical methods allow a reliable description of the system and its various physical properties, specifically in the context of the phase boundaries separating the compressible SF regime from the incompressible insulating regions.

The paper is organized as follows. In Sec. II we review the model at hand and present a qualitative description of its expected phase diagram. In Sec. III, we compute the phase 
boundaries between the SF phase and the empty and filled lattices analytically. In Sec. IV, we obtain the remaining boundary between the SF and the half-filled MI phase. This phase boundary is computed numerically, using the SSE algorithm. Section V is devoted to studying the phase diagram as it is given by two mean-field approaches and in Sec. VI we employ a strong-coupling expansion (SCE) method. These approximation methods are then compared against the previously obtained numerically exact results. In Sec. VII, we study the momentum distribution of the bosons, in order to allow for a comparison with future experimental data. Finally, in Sec. VIII, we conclude with a discussion and summary of our results.

\section{MODEL}

The Hamiltonian for hard-core bosons in a period-two hypercubic superlattice in $d$ dimensions, with $N=L^{d}$ sites and periodic boundary conditions, can be written as

$$
\hat{H}=-t \sum_{\langle i j\rangle}\left(\hat{a}_{i}^{\dagger} \hat{a}_{j}+\hat{a}_{j}^{\dagger} \hat{a}_{i}\right)-A \sum_{i}(-1)^{\sigma(i)} \hat{n}_{i}-\mu \sum_{i} \hat{n}_{i} .
$$

Here, $\langle i j\rangle$ denotes nearest neighbors, $\hat{a}_{i}\left(\hat{a}_{i}^{\dagger}\right)$ destroys (creates) a hard-core boson on site $i, \hat{n}_{i}=\hat{a}_{i}^{\dagger} \hat{a}_{i}$ is the local density operator, $\mu$ is the global chemical potential, and $A(-1)^{\sigma(i)}$ is a checkerboard local potential with $\sigma(i)=0$ on the even sublattice and 1 on the odd sublattice. The hopping parameter $t$ (which we shall fix at $t=1$ ) sets the energy scale and without loss of generality we choose $A>0$. The hardcore boson creation and annihilation operators satisfy the constraints $\hat{a}_{i}^{\dagger 2}=\hat{a}_{i}^{2}=0$ and $\left\{\hat{a}_{i}, \hat{a}_{i}^{\dagger}\right\}=1$, which prohibit double or higher occupancy of lattice sites, as dictated by the $U \rightarrow \infty$ limit of the Bose-Hubbard model. For any two different sites $i \neq j$, the creation and annihilation operators obey the usual bosonic relations $\left[\hat{a}_{i}, \hat{a}_{j}\right]=\left[\hat{a}_{i}^{\dagger}, \hat{a}_{j}^{\dagger}\right]=\left[\hat{a}_{i}, \hat{a}_{j}^{\dagger}\right]=0$.

To understand the zero-temperature phase diagram of hard-core bosons in a superlattice potential, let us first analyze the atomic $(t=0)$ limit. In this limit, there is no kinetic (hopping) term, and the boson number operators $\hat{n}_{i}$ commute with the Hamiltonian, so every lattice site is occupied by a fixed number of bosons. The average boson occupancy is determined so as to minimize the ground-state (free) energy. In particular, for $A=0$, the model is translationally invariant, and the ground-state boson occupancy is the same for each of the lattice sites: for $\mu<0$ the minimal energy configuration is simply the particle vacuum (VP), i.e., the completelyempty lattice, and for $\mu>0$ the minimal energy configuration is simply the hole vacuum (VH), i.e., the completelyfilled lattice. The ground-state energy of these phases is degenerate at $\mu=0$. When $A \neq 0$, the ground state has an additional half-filled insulating phase characterized by crystalline order in the form of staggered boson densities, i.e., $\left\langle\hat{n}_{i}\right\rangle=1$ for the even (or odd, depending on the sign of $\mu / A$ ) sublattice and $\left\langle\hat{n}_{i}\right\rangle=0$ for the odd (or even) one. We call this alternating density pattern the MI phase although it is sometimes referred to as a charge-density wave. ${ }^{20}$ The MI phase resides in the region $|\mu / A|<1$, sandwiched between the particle vacuum and the hole vacuum.

Having discussed the $t=0$ limit, we are now ready to analyze the competition between the kinetic- and the potential-

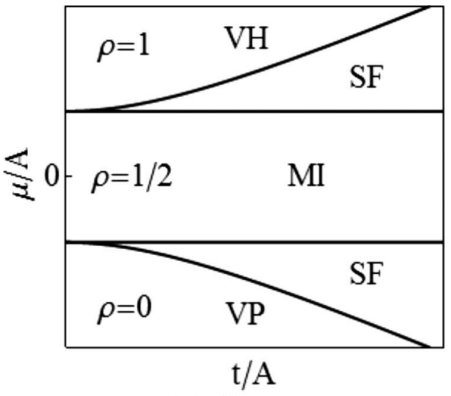

(a) $d=1$

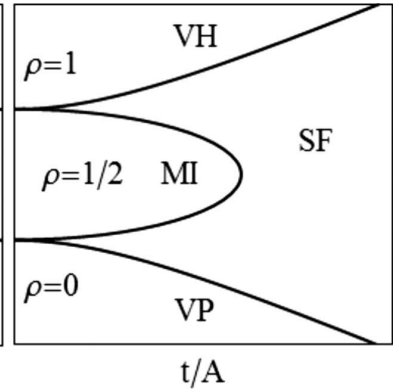

(b) $d>1$
FIG. 1. Phase diagram of the hard-core Bose-Hubbard model in the presence of a period-two superlattice, Eq. (1). In one dimension (left panel), the phase diagram contains three incompressible insulating phases, indicated by $\mathrm{VH}$ - the hole vacuum, i.e., a completely-filled lattice, VP-the particle vacuum, i.e., the completely-empty lattice, and MI-the Mott insulator, in which case the average density is $1 / 2$ and the local densities on the even and odd sublattices are different. Outside of these insulating regions, the system is SF. In higher dimensions (right panel), the phase diagram is similar, with one exception: while in one dimension the MI phase extends to infinity, in higher dimensions the MI phase takes the form of a Mott lobe.

energy terms of the Hamiltonian when $t \neq 0$. In one dimension, the phase diagram of the model is already known. As noted in the Introduction, the model in this case has an analytic solution. ${ }^{20,22}$ This is due to the Jordan-Wigner transformation which enables the mapping of the hard-core boson Hamiltonian to that of noninteracting spinless fermions. The dispersion relation in this case is given by

$$
\varepsilon(k)=-\mu \pm \sqrt{4 t^{2} \cos ^{2}(k a)+A^{2}},
$$

where $a$ is the lattice constant. The phase diagram consists of three insulating incompressible regions (these are extensions of the $t=0$ ones), as shown in Fig. 1(a). Two are the trivial insulators: the VP phase which is obtained for large and negative values of $\mu$, and the $\mathrm{VH}$ phase which is obtained for large and positive values of $\mu$. These two phases are also present in the absence of the alternating potential and are particle-hole "mirror images" of each other. They are separated from the SF phase along the curves $\mu / A= \pm \sqrt{1+(2 t / A)^{2}}$ [see Fig. 1(a)]. As evident from the expression for the dispersion relations given above, the superlattice (i.e., the onsite checkerboard potential) creates a gap of $\Delta=2 A$ in the energy spectrum, leading to a MI phase at half filling. This is the "slab" enclosed by $\mu / A=1$ from above and $\mu / A=-1$ from below, in the center of the figure.

In dimensions higher than one [Fig. 1(b)], the expected phase diagram of the hard-core Bose-Hubbard model is qualitatively similar to the one-dimensional case with one notable exception. Here, the MI region does not extend to infinity, but instead is a finite lobe, connecting the two SF regimes together.

The phase diagram of the hard-core Bose-Hubbard model has one additional property resulting from it being invariant under the transformation $\hat{a}_{i} \rightarrow \hat{a}_{i+\hat{r}}^{\dagger}$ (where $\hat{r}$ denotes a shift of one lattice step in any of the possible directions). This sym- 
metry operation, which can be immediately read off from the Hamiltonian, corresponds to a particle-hole exchange combined with swapping the odd and even sublattices. It leads to a $\mu \rightarrow-\mu$ symmetry in the phase diagram. We shall make use of this fact when we obtain the phase diagram in later sections. The special case of $\mu=0$ has been studied in Ref. 19 both in two and three dimensions.

Before moving on, we recall that the model at hand can also be viewed as the $X Y$ model of a spin-1/2 system. ${ }^{25,26}$ This is due to the mapping between bosonic operators and $S U(2)$ generators

$$
\hat{a}_{i}^{\dagger} \leftrightarrow \hat{S}_{i}^{+}, \quad \hat{a}_{i} \leftrightarrow \hat{S}_{i}^{-}, \hat{a}_{i}^{\dagger} \hat{a}_{i} \leftrightarrow \hat{S}_{i}^{z}+1 / 2 .
$$

With this mapping, the hard-core bosons Hamiltonian, Eq. (1), becomes that of the $X Y$ antiferromagnet with an alternating magnetic field applied along the $\hat{z}$ direction

$$
\hat{H}=-t \sum_{\langle i j\rangle}\left(\hat{S}_{i}^{+} \hat{S}_{j}^{-}+\hat{S}_{j}^{+} \hat{S}_{i}^{-}\right)-\sum_{i}\left[\mu+A(-1)^{\sigma(i)}\right]\left(\hat{S}_{i}^{z}+\frac{1}{2}\right) .
$$

This alternative representation will become handy in the next sections.

\section{VACUUM OF PARTICLES AND HOLES PHASE BOUNDARIES}

As it turns out, the phase boundary separating the SF phase from the insulating VH phase (henceforth, the SF-VH boundary) can be easily obtained analytically for any given dimension. To see this, we will use the fact that our Hamiltonian commutes with the total-number-of-bosons operator $\hat{N}=\sum_{i} \hat{n}_{i}$. In spin language, this simply means that for any given set of parameters $\mu, A$, and $t$, the ground-state wave function will be a linear combination of product states each having the same number of spin-downs. In the $\mathrm{VH}$ phase, this number is zero, as the wave function is simply

$$
|\mathrm{VH}\rangle=|\uparrow \uparrow \uparrow \cdots \uparrow \uparrow \uparrow\rangle
$$

with energy $\varepsilon_{\mathrm{vh}}=-\mu N$. In the infinitesimally thin layer outside the VH phase, the state of the system (which we shall refer to as the VH "defect" state) is characterized by exactly one spin-down. That is, the wave function has the form

$$
\left|\mathrm{VH}_{\mathrm{def}}\right\rangle=\sum_{i} c_{i} \hat{S}_{i}^{-}|\mathrm{VH}\rangle
$$

The symmetry of our model further tells us that all the coefficients $c_{i}$ whose index " $i$ " corresponds to a site on the even (odd) sublattice are all the same, namely,

$$
c_{i}=\frac{c_{\text {e.s. }}+c_{\text {o.s. }}}{2}+(-1)^{\sigma(i)} \frac{c_{\text {e.s. }}-c_{\text {o.s. }}}{2},
$$

where normalization requires $N / 2\left(\left|c_{\text {e.s. }}\right|^{2}+\left|c_{\text {o.s. }}\right|^{2}\right)=1$ and e.s. (o.s.) stands for the even (odd) sublattice. In order to determine the exact value of the weights $c_{\text {e.s. }}$ and $c_{\text {o.s. }}$, we first act with the Hamiltonian on this state. This eigenvalue problem then reduces to the following coupled equations:

$$
\begin{aligned}
& -2 d t c_{\text {o.s. }}+[\mu(1-N)+A] c_{\text {e.s. }}=\varepsilon c_{\text {e.s. }} \\
& -2 d t c_{\text {e.s. }}+[\mu(1-N)-A] c_{\text {o.s. }}=\varepsilon c_{\text {o.s. }}
\end{aligned}
$$

where $\varepsilon$ is the energy of the state. Solving for $\varepsilon$, the solution with minimal energy turns out to be

$$
\varepsilon_{\mathrm{def}}=-\mu N+\mu-\sqrt{A^{2}+(2 d t)^{2}} .
$$

The SF-VH boundary is the curve along which the VH state, Eq. (5), is no longer energetically favorable. This happens when its energy becomes equal to the energy of the defect state, Eq. (6). Matching the two, we obtain the SF-VH phase boundary

$$
\frac{\mu}{A}=\sqrt{1+x^{2}}
$$

where $x=2 d t / A$.

A few remarks are now in order. As already noted in the previous section, the phase diagram of the hard-core Bose-Hubbard model is symmetric under the transformation $\mu \rightarrow-\mu$. This tells us that the SF-VP phase boundary [the lowest branch in Fig. 1(b)], is given by $\mu / A=-\sqrt{1+x^{2}}$. This result can also be obtained by repeating the above exercise with the substitution $|\uparrow\rangle \leftrightarrow|\downarrow\rangle$. We also note that Eq. (10) agrees with the corresponding expression of the onedimensional case obtained formerly (see Sec. III).

Another, simpler argument leading to the same solution stems from the fact that the boundary between the SF and the VP (VH) phase is determined by the addition of a single particle (hole) to the completely-empty (-filled) lattice. It can then be argued that whether one is dealing with hard-core bosons or noninteracting spinless fermions, makes no difference in this case, as the particle statistics plays no role. This further means that one needs only to diagonalize the singleparticle Hamiltonian and find the energy difference between the completely-empty (-filled) lattice and the state with one particle (hole). These will provide the chemical potential at the boundary between the SF and the VP (VH) phase. The single-particle spectrum in a $d$-dimensional superlattice with period two has the form

$$
\varepsilon(k)=-\mu \pm \sqrt{4 d^{2} t^{2} \cos ^{2}(k a)+A^{2}}
$$

from which Eq. (10) follows trivially.

\section{NUMERICAL RESULTS}

Unlike the SF-VH and SF-VP phase boundaries, the SF-MI boundary, cannot be determined with the tools introduced in the previous section. One reason for that is that the exact many-body wave function of the MI state is not known. Therefore, in this section we explore the SF-MI phase boundary numerically by performing simulations based on the SSE algorithm. ${ }^{23,24}$ Our main objective here is to find the critical points of the SF-insulator transitions in the $\mu$ - $A$ parameter space (without loss of generality we fix the hopping parameter at $t=1$ and consider only $\mu>0$ and $A>0$ ). Critical points on the SF-MI boundary were typically obtained by first fixing the value of the parameter $A$, and then 


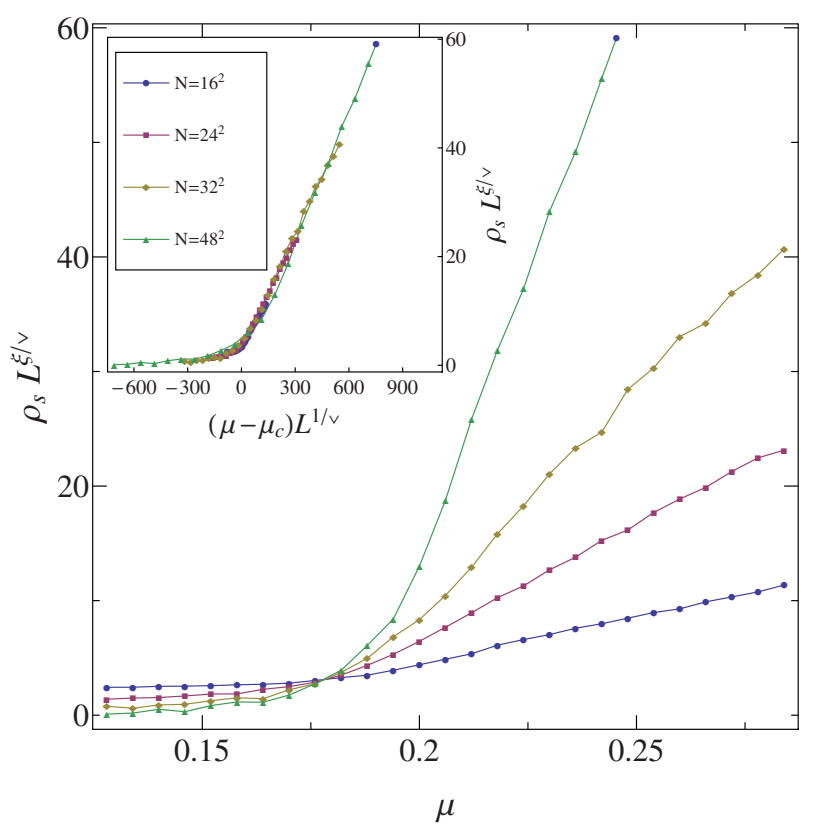

FIG. 2. (Color online) Scaled superfluid density as a function of the chemical potential $\mu$ for the various system sizes in the two-dimensional case (here, $A=1.05$ ). All the curves intersect at $\mu \approx 0.178$ indicating the value of the critical point. In the inset, the control parameter (the horizontal axis) is scaled as well, leading to the collapse of all data points into a single curve.

performing the simulations for a range of values of $\mu$ and different system sizes. This procedure was then repeated for different values of $A$. In some cases, mainly near the tip of the Mott lobe, we repeated the above procedure by fixing the value of $\mu$ and performing simulations for a range of $A$ values and different system sizes. This was done mainly to further verify the accuracy of the results, as the tip of the lobe is a multicritical point and therefore requires more care.

Repeating the simulations with different system sizes, enables us to extrapolate the thermodynamic limit by correcting finite-size effects using scaling arguments in the vicinity of the phase transition: around the critical point, most physical quantities (which we denote here by $X$ ) scale according to the general rule

$$
X L^{\xi / \nu}=F\left(\left|\mu-\mu_{\mathrm{c}}\right| L^{1 / \nu}\right),
$$

where $F$ is a universal scaling function, $\mu-\mu_{\mathrm{c}}$ is the shifted control parameter ( $\mu$ being the control parameter and $\mu_{\mathrm{c}}$ its critical value), $\nu$ is the correlation length critical exponent, and $\xi$ is the critical exponent belonging to the observable $X$. The values of these exponents are determined by the universality class the transition belongs to. In a previous work, ${ }^{19}$ we studied the SF-MI transition at fixed (half-filled) density. This type of transition belongs to the $(d+1) X Y$ universality class, similarly to the SF-MI transition of the Bose-Hubbard model at fixed integer density. ${ }^{5}$ Here, we compute the phase boundary between the SF and the (half-filled) MI phase while changing the density, so the transition belongs to the mean-field universality class for which the correlation length and dynamical critical exponents are $\nu=1 / 2$ and $z=2$ (again,

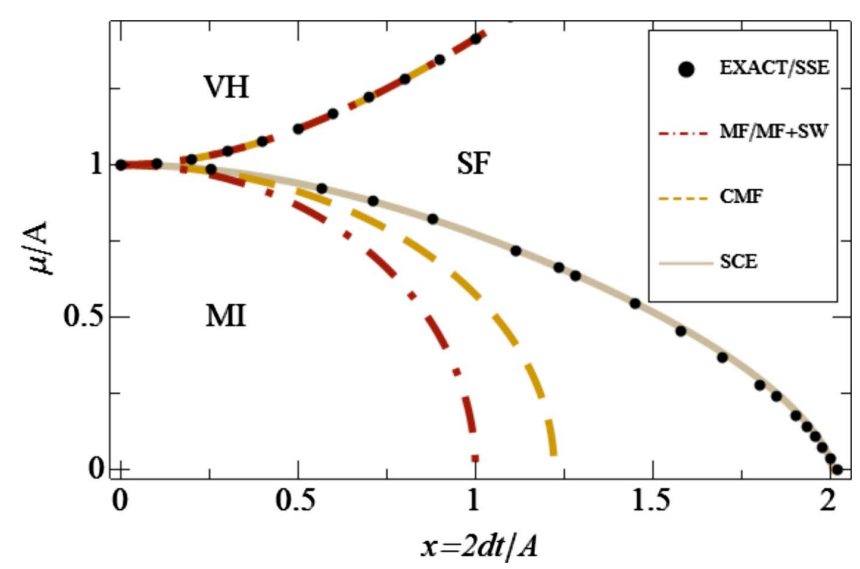

FIG. 3. (Color online) Phase diagram of the model in two dimensions. The full circles are the analytical (VH boundary) and numerical (MI boundary) results. The solid line corresponds to the SCE fit, whereas the dot-dashed and dashed lines are the mean-field (with and without spin-wave corrections) and cluster mean-field predictions, respectively. As the figure shows, the SF-VH boundary is predicted correctly by the mean-field approximation schemes. As for the SF-MI boundary, the predictions of the SCE fit provide the most accurate results.

exactly as the corresponding transition in the Bose-Hubbard model). ${ }^{5}$

Equation (12) above will help us find the critical point, as it tells us that (a) the quantity $X L^{\xi / \nu}$ should be independent of the size of the system at the phase transition and (b) when plotting $X L^{\xi / \nu}$ against $\left|\mu-\mu_{\mathrm{c}}\right| L^{1 / \nu}$ the resulting curve should be independent of the system size as well. The quantity we shall be using to that end is the superfluid density, which has the critical exponent $\xi=\nu(d+z-2)$ (see Ref. 5 for details) where $d$ is the dimension.

We note here that since we are interested in the zerotemperature properties of the system, simulations are performed with high inverse temperature $\beta=1 / T$ (in our units, $k_{B}=1$ ), where in most cases we will find it sufficient to have $\beta \geq 2 L$ in order to obtain virtually zero-temperature results. (The effects of increasing $\beta$ beyond this value are indiscernible.)

As already discussed, in one dimension, our model has an analytic solution. ${ }^{20}$ This enabled us to compare our numerical method against exact analytic results, as a check on our computational approach. No discrepancies between the analytical solution and the numerical one were found (see also Ref. 19).

In dimensions higher than one, no analytic solution to the model exists, so accurate results are obtainable only numerically. In the two-dimensional case, we have applied the SSE algorithm to systems of sizes ranging from $16 \times 16$ to 48 $\times 48$, with inverse temperature $\beta=64$. Figure 2 is an example of how scaling of the superfluid density data for the various system sizes is performed in order to find the critical point corresponding to $A=1.05$. Here, the scaled superfluid density is plotted against $\mu$ for the different system sizes (the statistical errors of the quantum Monte Carlo simulations are on the order of magnitude of the symbol sizes). All curves intersect at $\mu_{\mathrm{c}} \approx 0.178$, signifying the phase transition for 


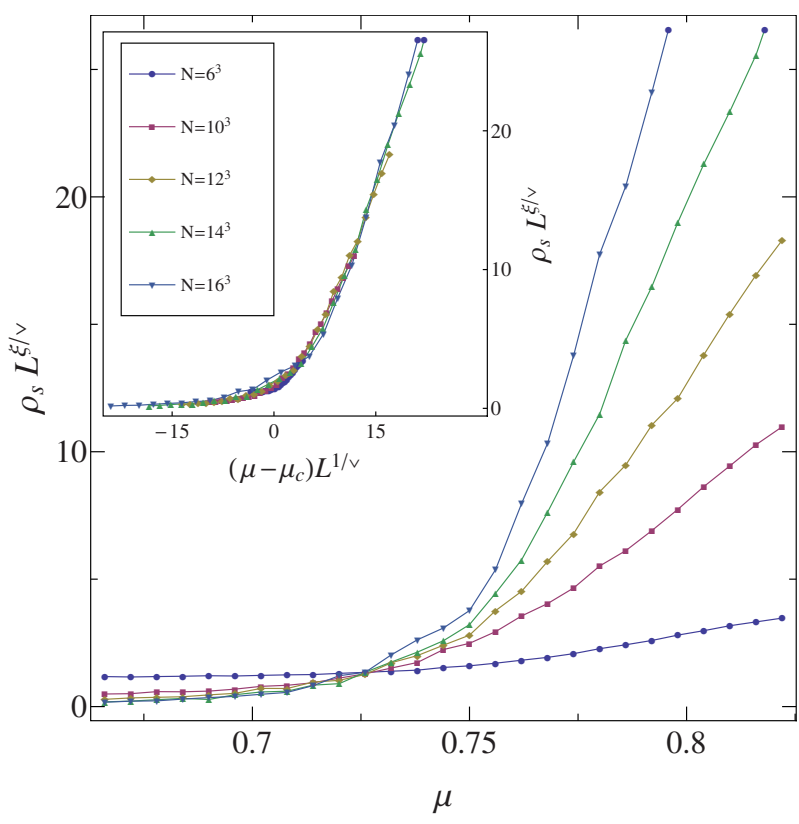

FIG. 4. (Color online) Scaled superfluid density as a function of the chemical potential $\mu$ for the various system sizes in the threedimensional case (here, $A=2.28$ ). All the curves intersect at $\mu \approx 0.725$ indicating the value of the critical point. In the inset, the control parameter (the horizontal axis) is scaled as well, leading to the collapse of all data points into a single curve.

$A=1.05$. The inset shows the scaled superfluid density as a function of the scaled control parameter, in which case all curves should be, and in fact are, on top of each other. The resulting SF-MI phase boundary of our model in two dimensions is marked by the full circles in Fig. 3. As noted earlier, the lower half of the phase diagram Fig. 1 (the $\mu<0$ half) is but a mirror image of the portion shown in Fig. 3 and thus is

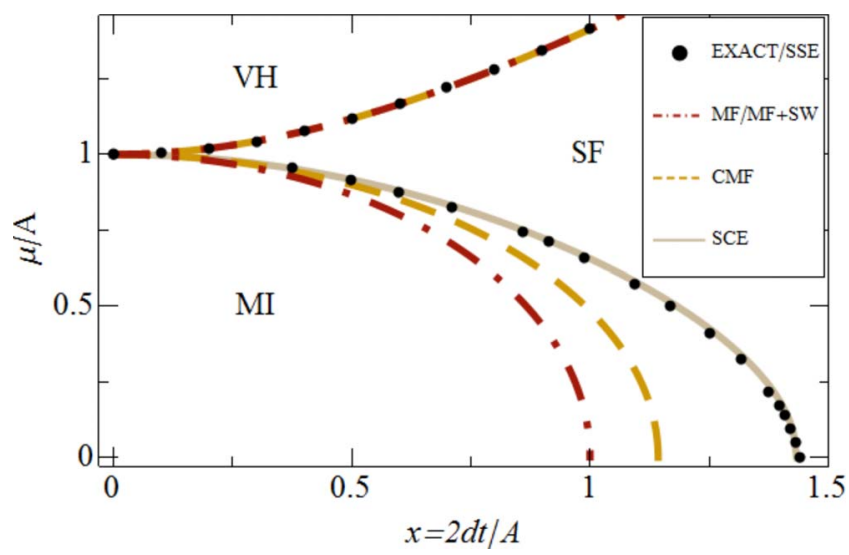

FIG. 5. (Color online) Phase diagram of the model in three dimensions. The full circles are the analytical (VH boundary) and numerical (MI boundary) results. The solid line corresponds to the SCE fit, whereas the dot-dashed and dashed lines are the mean-field (with and without spin-wave corrections) and cluster mean-field predictions, respectively. As the figure shows, the SF-VH boundary is predicted correctly by the mean-field approximation schemes. As for the SF-MI boundary, the predictions of the SCE fit provide the most accurate results. not presented there. The tip of the Mott lobe was found to be at $x_{c} \approx 2.02 .{ }^{19}$

In three dimensions, we have performed simulations with system sizes ranging from $6 \times 6 \times 6$ to $16 \times 16 \times 16$ and an inverse temperature of $\beta=40$. Figure 4 is an example of how scaling is carried out in three dimensions: the scaled superfluid density is plotted as a function of $\mu$ for the different system sizes and $A=2.28$. The inset depicts the scaled superfluid density as a function of the scaled control parameter, exhibiting the collapse of all data points into a single curve, as in two dimensions. The resulting phase boundary in three dimensions is shown in Fig. 5 (full circles). The tip of the Mott lobe was found to be at $x_{c} \approx 1.44$. $^{19}$

\section{MEAN-FIELD APPROACHES}

Having obtained the exact boundaries of the phase diagram of the model, we now proceed to study several approximation schemes and examine the extent to which they provide an accurate description of the phase diagram of the model. We start this investigation with the Gutzwiller meanfield approach.

\section{A. Gutzwiller mean-field}

Along the lines of Ref. 19, we start our mean-field calculation with the following product state as our ansatz:

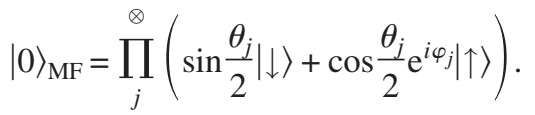

The angles $\left(\theta_{j}, \varphi_{j}\right)$ here, specify the orientation of the $j$ th spin. Naturally, we expect the wave functions of each of the odd (even) sublattice sites to be identical. This is due to the checkerboard symmetry of the model.

As we are using the grand-canonical scheme, the orientations of the spins will be determined by minimizing the grand-canonical potential (per site)

$$
\begin{aligned}
\Omega_{\mathrm{MF}}= & { }_{\mathrm{MF}}\langle 0|\hat{H}| 0\rangle_{\mathrm{MF}} \\
= & -\frac{t}{2 N} \sum_{\langle i j\rangle} \sin \theta_{i} \sin \theta_{j} \cos \left(\phi_{i}-\phi_{j}\right) \\
& -\frac{1}{2 N} \sum_{i}\left[\mu+A(-1)^{\sigma(i)}\right]\left(1+\cos \theta_{i}\right)
\end{aligned}
$$

with respect to these angles. For the azimuthal angles, this simply implies a constant (yet arbitrary) value $\varphi_{j}=\Phi$, while for the polar angles, the minimizers are

$$
\begin{aligned}
& \cos \theta_{1}=\operatorname{Min}\left[1, \operatorname{Max}\left[-1, \mu_{1} \sqrt{\frac{1+\mu_{2}^{2}}{1+\mu_{1}^{2}}}\right]\right], \\
& \cos \theta_{2}=\operatorname{Min}\left[1, \operatorname{Max}\left[-1, \mu_{2} \sqrt{\frac{1+\mu_{1}^{2}}{1+\mu_{2}^{2}}}\right]\right],
\end{aligned}
$$

where $\mu_{1,2}=(\mu \pm A) /(2 d t)$. We note that while in Ref. 19 the focus was on the special $\mu=0$ case, here we place no limitations on $\mu$. 
At this point we can calculate the following quantities. First, the average density of particles is:

$$
\begin{aligned}
\rho_{\mathrm{MF}} & =\frac{1}{N} \sum_{i}{ }_{\mathrm{MF}}\left\langle 0\left|\hat{a}_{i}^{\dagger} \hat{a}_{i}\right| 0\right\rangle_{\mathrm{MF}} \\
& =\frac{1}{2}+\frac{1}{2 N} \sum_{i} \cos \theta_{i} \\
& =\frac{1}{2}+\frac{1}{4}\left(\cos \theta_{1}+\cos \theta_{2}\right) .
\end{aligned}
$$

Next, the free energy becomes

$$
\begin{aligned}
\Omega_{\mathrm{MF}}= & { }_{\mathrm{MF}}\langle 0|\hat{H}| 0\rangle_{\mathrm{MF}} \\
= & -\frac{d t}{2} \sin \theta_{1} \sin \theta_{2}-\frac{\mu}{2}-\frac{1}{4}(\mu+A) \cos \theta_{1} \\
& -\frac{1}{4}(\mu-A) \cos \theta_{2}
\end{aligned}
$$

and the density of bosons in the zero-momentum mode $\rho_{0}$ is calculated as

$$
\begin{aligned}
\rho_{0, \mathrm{MF}} & =\frac{1}{N^{\mathrm{MF}}}\left\langle 0\left|\hat{a}_{k=0}^{\dagger} \hat{a}_{k=0}\right| 0\right\rangle_{\mathrm{MF}} \\
& =\frac{1}{4 N^{2}} \sum_{i, j} \sin \theta_{i} \sin \theta_{j} \\
& =\frac{1}{16}\left(\sin \theta_{1}+\sin \theta_{2}\right)^{2} .
\end{aligned}
$$

The superfluid density too is obtained in a straightforward manner. In the mean-field approximation it has the simple form $\rho_{s}=-(2 d)^{-1} \partial \Omega / \partial t$. $^{19}$

The phase boundaries are simply the curves along which the superfluid density and the zero-momentum fraction drop to zero. These turn out to be

$$
\frac{\mu}{A}=\sqrt{1 \pm x^{2}}
$$

where the "+" branch belongs to the SF-VH transition and the "-" branch belongs to the SF-MI transition (again, $x$ $=2 d t / A)$. The phase diagram of the model as predicted by the Gutzwiller mean-field approach is sketched in Fig. 6, which shows the average density of bosons as a function of $x$ and $\mu / A$.

An alternative way of deriving the mean-field phase boundaries is through the decoupling approximation. ${ }^{5,27}$ In this approach, one approximates the hopping term as

$$
\hat{a}_{i}^{\dagger} \hat{a}_{j} \approx\left\langle\hat{a}_{i}^{\dagger}\right\rangle \hat{a}_{j}+\hat{a}_{i}^{\dagger}\left\langle\hat{a}_{j}\right\rangle-\left\langle\hat{a}_{i}^{\dagger}\right\rangle\left\langle\hat{a}_{j}\right\rangle
$$

and introduces the condensate order parameter $\psi_{i}=\sqrt{\hat{n}_{i}}=\left\langle\hat{a}_{i}^{\dagger}\right\rangle=\left\langle\hat{a}_{i}\right\rangle$ (analogous to the Bogoliubov approach). Since the condensate order parameter is the same for all lattice sites belonging to the same sublattice, i.e.,

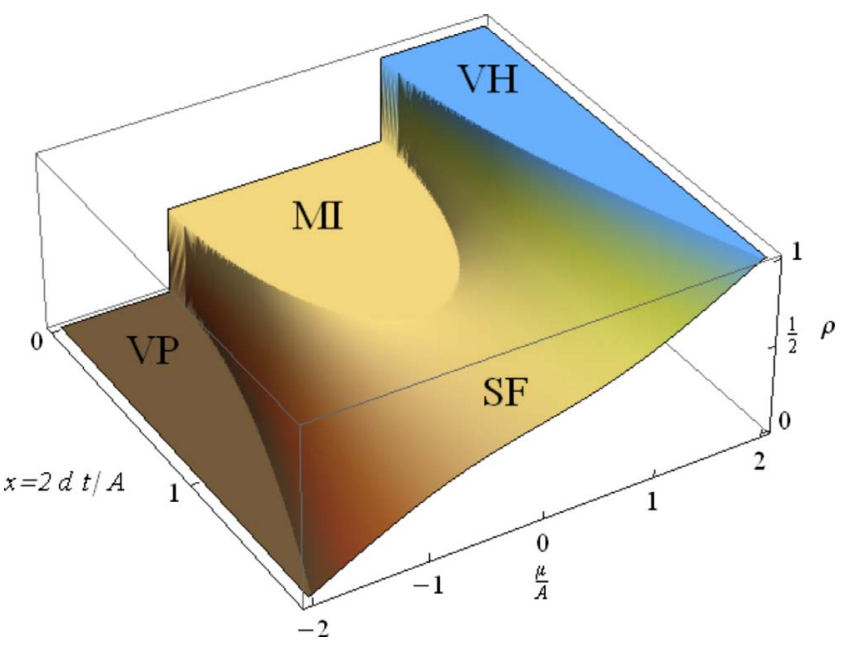

FIG. 6. (Color online) Average density of bosons as a function of $x=2 d t / A$ and $\mu / A$ in the mean-field approximation. The three insulating phases VP (empty lattice, zero density), MI (half-filled lattice), and VH (completely-filled lattice) are seen very clearly in the figure. Outside of these insulating regions is the SF phase.

$$
\psi_{i}=\frac{\psi_{\text {e.s. }}+\psi_{\text {o.s. }}}{2}+(-1)^{\sigma(i)} \frac{\psi_{\text {e.s. }}-\psi_{\text {o.s. }}}{2}
$$

for some real unknown parameters $\psi_{\text {e.s. }}$ and $\psi_{\text {o.s. }}$ (due to the checkerboard symmetry of the model), it is sufficient to solve only for the effective two-site Hamiltonian

$$
\begin{aligned}
\hat{H}^{\mathrm{MF}}= & -2 d t \psi_{\mathrm{e} . \mathrm{s} .}\left(\hat{a}_{j}^{\dagger}+\hat{a}_{j}\right)-2 d t \psi_{\mathrm{o} . \mathrm{s} .}\left(\hat{a}_{i}^{\dagger}+\hat{a}_{i}\right) \\
& +4 d t \psi_{\mathrm{e} . \mathrm{s} .} \psi_{\mathrm{o} . \mathrm{s} .}-A \hat{n}_{i}+A \hat{n}_{j}-\mu\left(\hat{n}_{i}+\hat{n}_{j}\right),
\end{aligned}
$$

where $i \in$ e.s. and $j \in$ o.s. Performing a second-order perturbation theory in the first two terms of this effective Hamiltonian around the $\mathrm{VH}$ and $\mathrm{MI}$ phases produces the groundstate energies as a function of $\psi_{\text {e.s. }}$ and $\psi_{\text {o.s. }}$. Notice that higher orders are not needed for our purposes since the second-order theory is sufficient to derive the energy functional of the system up to second order in the order parameters $\psi_{\mathrm{oss}}$ and $\psi_{\text {e.s. }}$. Following the usual Landau procedure for second-order phase transitions, minimizing the groundstate energies as a function of the superfluid order parameters, we eventually arrive at Eq. (19).

The dash-dotted lines in Figs. 3 and 5 show the phase diagram as predicted by the Gutzwiller mean-field approach, compared against the numerical results. Interestingly, the mean-field ansatz yields the correct solution for the SF-VH transition (upper branch). On the other hand, for the SF-MI boundary, mean-field results differ considerably from the numerical data: while away from the tip of the Mott lobe the method is very accurate, as one approaches the tip itself, errors climb up to their maximal values of $\approx 100 \%$ in two dimensions and $\approx 50 \%$ in three dimensions at the tip of the MI lobe. The very large errors here reflect the fact that the mean-field approach is simply not fit to describe the phase transition in this region. 
Before moving on, we remark here that addition of spinwave corrections to the mean-field solution does not modify the mean-field critical points of the model ${ }^{19}$ so the phase boundary is not altered by spin-wave corrections. While deep in the SF phase spin-wave corrections yield major improvements over the mean-field results for many of our observables of interest, in terms of phase boundaries the spin-wave corrections do not contribute. As one approaches the phase transition itself, the spin-wave corrections lose their accuracy, eventually leaving the phase boundaries at their meanfield values. ${ }^{19}$

\section{B. Cluster mean field}

Aiming to improve the results obtained in the previous section, we now describe a "cluster" mean-field approach, which makes use of the checkerboard symmetry of the model. This approximation scheme was introduced in Ref. 19 where it was applied to the special case of $\mu=0$. Within this approach, one starts with a variational ansatz which, as before, is a product state. However, this time one does not choose a product of single-site wave functions. The new ansatz is a product of wave functions each describing the state of a "block" of $2^{d}$ sites, such that with this block as the basic cell, the model turns homogeneous. In two dimensions, for example, a block is a $2 \times 2$ square cell, and the system is described by the general wave function

$$
|0\rangle_{\mathrm{CMF}}=\prod_{\text {blocks }}^{\otimes}\left(\sum_{i, j, k, l \in\{\downarrow, \uparrow\}} c_{i j k l}|i j k l\rangle\right),
$$

where the generalization to three dimensions, in which case the basic block is a $2 \times 2 \times 2$ cubic cell, is straightforward (note that the coefficients for each of the blocks will be the same due to the symmetry of the wave function). ${ }^{19}$ As before, we minimize the free energy $\Omega_{\mathrm{CMF}}={ }_{\mathrm{CMF}}\langle 0|\hat{H}| 0\rangle_{\mathrm{CMF}}$ with respect to the coefficients $c_{i j k l}$ of the wave function (this time we do so numerically). Obtaining the various observables in terms of the wave function given in Eq. (23) is straightforward and was performed in much the same way as the usual mean-field approach discussed in Sec. V A.

The phase boundaries, as predicted by the cluster meanfield approximation, are given by the dashed lines in Figs. 3 and 5 for two and three dimensions, respectively. As the figures indicate, the SF-VH boundary is predicted correctly. This is no surprise as the Gutzwiller mean-field, over which the current method is an improvement, is already exact for that boundary. As for the SF-MI boundary, the cluster meanfield method is far better than the Gutzwiller mean-field method. As in the previous mean-field case, the results are more accurate away from the tip of the Mott lobe but reach $\approx 60 \%$ error in two dimensions $\approx 24 \%$ error in three dimensions, as the tip is approached.

Having shown that the mean-field-type theories presented here are not very accurate in describing the SF-MI phase boundary, in particular, close to the tip of the lobe, we turn to develop a strong-coupling perturbation theory in the hopping $t$. This approach, combined with a scaling analysis, will allow us to predict the critical point and the shape of the insulating lobe in a more accurate manner.

\section{SCE}

SCE techniques were previously used to discuss the phase diagram of the Bose-Hubbard model,,${ }^{8,9,28}$ and of the extended Bose-Hubbard model, ${ }^{29}$ and its results showed an excellent agreement with quantum Monte Carlo simulations ${ }^{11,12}$ in the former case. Motivated by the success of this technique with Bose-Hubbard-type models, here we generalize this technique to the hard-core Bose-Hubbard model on a superlattice.

To determine the phase boundary separating the incompressible MI phase from the compressible SF phase within the SCE method, one needs the energy of the MI phase and its defect states-those states which have one flipped spin (equivalently, one excited particle) about the groundstate-as a function of the parameter $t$. At the point where the energy of the incompressible state becomes equal to its defect state, the system becomes compressible, assuming that the compressibility approaches zero continuously at the phase boundary. Note that these arguments are very similar to those presented in Sec. III where exact results were obtained for the SF-vacuum insulators boundaries. Here however, the state of the system inside the MI phase is not known except for the special case $t=0$, where

$$
\left|\mathrm{MI}^{(0)}\right\rangle=|\uparrow \downarrow \uparrow \downarrow \cdots \uparrow \downarrow \uparrow \downarrow\rangle,
$$

where all the spin-ups (spin-downs) belong to the even (odd) sublattice.

The energy of the MI phase is calculated via a many-body version of the nondegenerate Rayleigh-Schrödinger perturbation theory up to fourth order in $t$. We note that all odd-order terms in $t$ vanish for the $d$-dimensional hypercubic lattices considered in this manuscript. This is because this state cannot be connected to itself by only one hopping but rather requires two hoppings to be connected.

Calculation of the wave functions and energies for the defect states is more involved as it requires the use of the many-body version of the degenerate Rayleigh-Schrödinger perturbation theory. The reason for that lies in the fact that when exactly one extra particle is added to the MI phase, it could go to any of the N/2 lattice sites that belong to the odd sublattice since all of those states share the same energy when $t=0$ (recall that $N$ is the number of lattice sites). Therefore, the initial degeneracy of the MI defect state is of order $N / 2$. This degeneracy is lifted at second order in $t$, since all of the defect states occupy one of the sublattices, and they cannot be connected by one hopping, but rather require two hoppings to be connected. The wave function (to zeroth order in $t$ ) of the particle-defect state turns out to be

$$
\left|\mathrm{MI}_{\mathrm{def}}^{(0)}\right\rangle=\sum_{i \in \text { o.s. }} f_{i} \hat{S}_{i}^{+}\left|\mathrm{MI}^{(0)}\right\rangle,
$$

where $f_{i}$ is the eigenvector of the matrix $T_{i i^{\prime}}=\sum_{j \in \text { e.s. }} t_{i j} t_{j i^{\prime}}$ with the highest eigenvalue, such that $\Sigma_{i^{\prime} \in \text { o.s. }} T_{i i^{\prime}} f_{i^{\prime}}=4 d^{2} t^{2} f_{i}$. Here, $t_{i j}=t$ for $\langle i j\rangle$ and zero otherwise. The normalization condition requires that $\sum_{i \in \text { o.s. }}\left|f_{i}\right|^{2}=1$. The eigenvector with the highest eigenvalue corresponds to the lowest energy state, i.e., to the ground state. We calculate the energy of the $\left|\mathrm{MI}_{\mathrm{def}}^{(0)}\right\rangle$ phase via degenerate perturbation theory up to fourth 
order in $t$. Here too all odd-order terms in $t$ vanish.

A lengthy but straightforward calculation leads to the following expression for the SF-MI boundary (for further details regarding the calculation, we refer the reader to a similar calculation given in Ref. 29)

$$
\frac{\mu}{A}=1-\frac{d-1}{2 d} x^{2}-\frac{(d-1)(d-3)}{8 d^{2}} x^{4}+O\left(x^{6}\right),
$$

where $x=2 d t / A$. This expression is exact for all $d$-dimensional hypercubic lattices up to the given order. In one dimension, it agrees with the analytical solution ${ }^{20}$ of the model given by $\mu / A=1$ (see Sec. II). In the $d \rightarrow \infty$ limit, where the exact result is given by the mean-field expression, i.e., $\mu / A=\sqrt{1-x^{2}}$, Eq. (26) is the correct power-series expansion about $x=0$.

In the two- and three-dimensional cases, fourth-order SCE is not very accurate near the tip of the MI lobe, as the variable $x$ is not very small there. Therefore, an extrapolation technique is desirable in order to determine the phase boundary more accurately. Such an extrapolation is possible for the MI phase since it is already known for $d>1$ that the critical point at the tip of the MI lobe has the scaling behavior of a $(d+1) X Y$ model. Therefore, we propose the following ansatz for the MI lobe which includes the known power-law critical behavior of the tip of the lobe:

$$
\frac{\mu}{A}=\alpha_{0}\left(1+\alpha_{1} x+\alpha_{2} x^{2}+\alpha_{3} x^{3}+\alpha_{4} x^{4}\right) \times\left(x_{c}-x\right)^{z \nu},
$$

where $x_{c}=2 d t / A_{c}$ is the critical point which determines the location of the MI lobe tip and $z \nu$ is the critical exponent for the $(d+1) X Y$ model which determines the shape of the MI lobe near $x_{c}$. The parameters $\alpha_{i}$ are determined by matching Eq. (26) with Eq. (27), after the latter is expanded out to fourth order in $t$. This procedure leads to

$$
\begin{gathered}
\alpha_{0}=\frac{1}{x_{c}^{z \nu}}, \\
\alpha_{1}=\frac{z \nu}{x_{c}}, \\
\alpha_{2}=\frac{z \nu(z \nu+1)}{2 x_{c}^{2}}+e_{2} \\
\alpha_{3}=\frac{z \nu(z \nu+1)(z \nu+2)}{6 x_{c}^{3}}+\frac{z \nu}{x_{c}} e_{2} \\
\alpha_{4}=\frac{z \nu(z \nu+1)(z \nu+2)(z \nu+3)}{24 x_{c}^{4}}+\frac{z \nu(z \nu+1)}{2 x_{c}^{2}} e_{2}+e_{4},
\end{gathered}
$$

where $e_{2}=-(d-1) /(2 d)$ and $e_{4}=-(d-1)(d-3) /\left(8 d^{2}\right)$ are the coefficients of the second- and fourth-order terms in our SCE.

In our extrapolations, we set $z \nu \approx 0.672$ for $d=2$ and $z \nu=1 / 2$ for $d>2$. This leaves only $x_{c}$ to be fixed; something which is accomplished by a straightforward $\chi^{2}$ curve fitting to the numerical data obtained in Sec. IV. The results are shown by the solid lines in Figs. 3 and 5 for two and three dimensions, respectively. As one can immediately see, the SCE results are very accurate and provide an analytic expression for the phase boundaries.

Alternatively, we can estimate $x_{c}$ using the above approach without fitting it to the numerical data. We do so by finding the value of $x_{c}$ for which the fifth-order term in $x$ of Eq. (27) vanishes. This gives $x_{c} \approx 1.53$ for $d=3(\approx 6.7 \%$ error) and $x_{c} \approx 1.076$ for $d \rightarrow \infty(\approx 7.6 \%$ error $)$.

Before moving on to the next section, we note here that a similar application of the SCE for the SF-VH phase boundary, where

$$
\left|\mathrm{VH}_{\mathrm{def}}^{(0)}\right\rangle=\sum_{i \in \text { o.s. }} f_{i} \hat{S}_{i}^{-}|\mathrm{VH}\rangle
$$

is the wave function (to zeroth order in $t$ ) of the hole-defect state, leads to

$$
\frac{\mu}{A}=1+\frac{1}{2} x^{2}-\frac{1}{8} x^{4}+O\left(x^{6}\right)
$$

in agreement with the exact result derived in Sec. III, i.e., $\mu / A=\sqrt{1+x^{2}}$, up to the given order. In addition, we perform a SCE in $A$, and find that the large $x$ behavior of the phase boundary is given by $\mu / A=x+O(1 / x)$, which is also in agreement with the exact result.

\section{MOMENTUM DISTRIBUTION}

Having discussed the phase diagram of the hard-core Bose-Hubbard model with a superlattice in the previous sections, next we analyze the momentum distribution $n(\mathbf{k})$ of these bosons. This quantity can be directly probed in experiments with ultracold atomic gases via an absorption imaging during a short time-of-flight. ${ }^{3,30}$ Since it is trivial to show that $n_{\mathrm{VH}}(\mathbf{k})=1$ in the $\mathrm{VH}$ phase, we shall concentrate only on the momentum distribution of the bosons in the MI phase, $n_{\mathrm{MI}}(\mathbf{k})$, where we will compare our numerical quantum Monte Carlo results with those of two analytical approaches: the random-phase approximation (RPA) and the SCE method introduced in the previous section.

The RPA is a well-defined linear operation in which thermal averages of products of operators are replaced by the product of their thermal averages. ${ }^{31}$ Since the fluctuations are not fully taken into account in this method, it becomes exact only for infinite-dimensional bosonic systems, recovering the mean-field theory. This method has been recently applied to the onsite, ${ }^{32,33}$ and extended ${ }^{34}$ Bose-Hubbard models, and its results showed good qualitative agreement with the experiments in the former case. ${ }^{3,30}$ Here we apply this method to our model (for further details regarding the calculation, we refer the reader to a similar calculation given in Ref. 34), and obtain

$$
n_{\mathrm{MI}-\mathrm{RPA}}\left(\varepsilon_{\mathbf{k}}\right)=\frac{1}{2} \sqrt{\frac{A-\varepsilon_{\mathbf{k}}}{A+\varepsilon_{\mathbf{k}}}},
$$

where $\varepsilon_{\mathbf{k}}=-2 t \sum_{i=1}^{d} \cos \left(k_{i} a\right)$ is the energy dispersion of noninteracting particles. Since the RPA phase boundary is exactly 
the same as the mean-field one and it gives a critical value for $x=2 d t / A$ that is much smaller than the true critical value in finite-dimensions, we compare our results with a rescaled $x$ value such that

$$
n_{\mathrm{MI}-\mathrm{RPA}}^{\text {scaled }}\left(\varepsilon_{\mathbf{k}}\right)=\frac{1}{2} \sqrt{\frac{A x_{c}-\varepsilon_{\mathbf{k}}}{A x_{c}+\varepsilon_{\mathbf{k}}}},
$$

where $x_{c}=2 d t / A_{c}$ is the true critical point which determines the location of the MI lobe tip. We call this the scaled RPA momentum distribution following Ref. 28.

To extend the RPA result to finite dimensions, we also calculate $n_{\mathrm{MI}}\left(\varepsilon_{\mathbf{k}}\right)$ as a power series expansion in the hopping $t$ via the strong-coupling perturbation theory. To second order in $t$, we obtain (for further details regarding the calculation, we again refer the reader to a similar calculation given in Ref. 34)

$$
n_{\mathrm{MI}-\mathrm{SCE}}\left(\varepsilon_{\mathbf{k}}\right)=\frac{1}{2}-\frac{\varepsilon_{\mathbf{k}}}{2 A}+\frac{\varepsilon_{\mathbf{k}}^{2}-2 d t^{2}}{4 A^{2}}+O\left(t^{3}\right),
$$

which is exact up to the given order for any dimension $d$. In the $d \rightarrow \infty$ limit (while $d t$ is kept fixed), we checked that Eq. (33) agrees with the RPA solution (which is exact in that limit) given in Eq. (31), when the latter is expanded out to second order in $t$. This provides an independent check of the algebra.

The second-order SCE is not very accurate near the tip of the MI lobe, as $t / A$ is not small there. To extend its region of validity, we therefore propose the following ansatz:

$$
n_{\mathrm{MI}}\left(\varepsilon_{\mathbf{k}}\right)=\frac{1}{2} \sqrt{\frac{A-\varepsilon_{\mathbf{k}}+(4 \lambda-2) d t^{2} / A}{A+\varepsilon_{\mathbf{k}}+4 \lambda d t^{2} / A}}
$$

for any dimension $d$, where $\lambda=d\left(x_{c}-1\right) / x_{c}^{2}$ depends on $d$. This expression reduces to Eq. (31) in the $d \rightarrow \infty$ limit and it has the correct power-series expansion about $x=0$ up to second order in $t$, i.e., Eq. (33). We call this the scaled SCE momentum distribution.

In Fig. 7, we show several comparisons (two in two dimensions and two in three dimensions) between the momentum distribution function obtained with the quantum Monte Carlo and the three approximations obtained above, namely, the scaled RPA, the SCE, and the scaled SCE. As the figures indicate, the scaled SCE is a far better fit than the other two methods, and more so for larger values of $A$, that is, deeper inside the MI phase where the SCE becomes more and more accurate.

\section{CONCLUSIONS}

We have obtained the complete phase diagram of the hard-core Bose-Hubbard model with a period-two superlattice in two and three dimensions. First we have calculated the boundaries between the superfluid phase and the trivial insulators (the completely-empty and completely-filled lattices) analytically. Then, using quantum Monte Carlo simulations followed by a finite-size scaling, we have determined the phase boundary between the superfluid phase and the (half-filled) Mott insulator. We have also compared our nu-
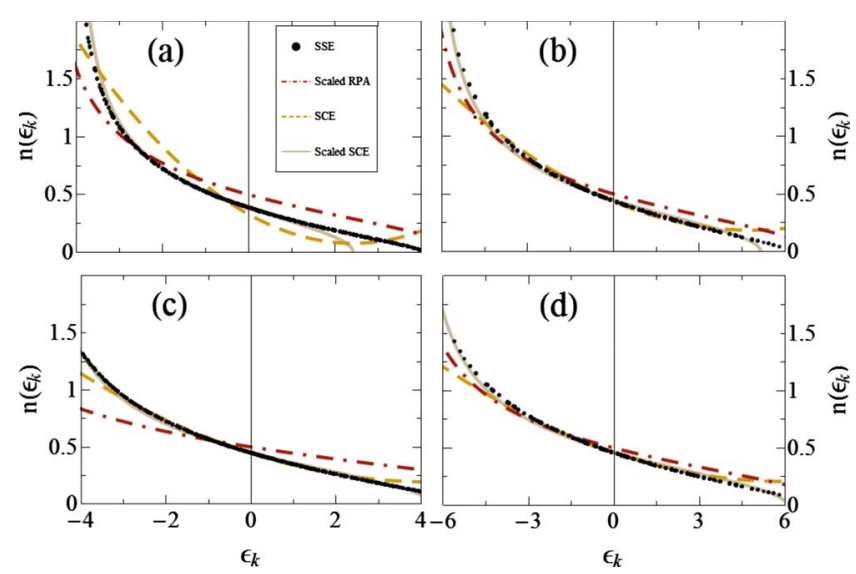

FIG. 7. (Color online) Momentum-distribution function $n\left(\varepsilon_{k}\right)$ for two $48 \times 48$ systems: (a) $A=2.42(x \approx 1.653)$ and (c) $A=4.22$ $(x \approx 0.948)$ and two $14 \times 14 \times 14$ systems: (b) $A=4.8(x=1.25)$ and (d) $A=6(x=1)$. The full circles are the numerical SSE results. The scaled RPA is the dot-dashed line, whereas the dashed and solid lines are the SCE and scaled SCE, respectively. The figures show that the scaled SCE results are much better than any of the other two approximation methods, and that the scaled SCE results fit better, as $A$ becomes larger ( $t=1$ in all four systems) — suggesting we are deeper inside the MI phase.

merical results against three approximation schemes: the usual Gutzwiller mean-field approach, a cluster mean-field approach, and the SCE method.

For the transition between the superfluid phase and the trivial completely-empty and completely-filled lattice insulators, we have found that the mean-field approaches yield the exact results in any dimension. As for the superfluid-Mott insulator boundary, the Gutzwiller approach was shown to work very poorly (up to $\approx 100 \%$ error in two dimensions and $\approx 50 \%$ error in three dimensions). This is a clear indication of the fact that this mean-field approach is not suitable for describing the superfluid-Mott insulator transition in the vicinity of the tip of the lobe. A cluster mean-field approximation scheme, which is based on the underlying checkerboard symmetry of the problem, was proven to be a big improvement over the previous method (reducing the error to one half of the one generated by the usual Gutzwiller ansatz), albeit still far from being accurate as one approaches the tip of the Mott lobe. The fourth-order SCE turned out to be the best method among the three in describing the superfluidMott insulator phase boundary, as the one-parametric fit of the SCE yielded very accurate results, also near the tip of the Mott lobe where the other methods failed. It also provided an analytic expression for that boundary, which could be used as a guide in future experimental realizations of this model.

Finally we have examined the extent to which several approximation schemes, such as the random phase approximation and the strong-coupling expansion, give an accurate description of the momentum distribution of the bosons inside the insulating phases. We have shown that a scaled SCE provides an accurate analytic expression for the momentum distribution of the bosons inside the Mott-insulating phase both in two and three dimensions, which could again be used as a guide in future experimental realizations of this model. 


\section{ACKNOWLEDGMENTS}

I.H and M.R. were supported by the U.S. Office of Naval Research under Grant No. N000140910966, and M.I. thanks
The Scientific and Technological Research Council of Turkey (TÜBITTAK) for financial support. We are grateful to J. K. Freericks and H. R. Krishnamurthy for useful discussions. *itayhe@physics.georgetown.edu

$\dagger$ miskin@ku.edu.tr

†mrigol@physics.georgetown.edu

${ }^{1}$ M. Greiner, O. Mandel, T. Esslinger, T. W. Hänsch, and I. Bloch, Nature (London) 415, 39 (2002).

${ }^{2}$ T. Stöferle, H. Moritz, C. Schori, M. Köhl, and T. Esslinger, Phys. Rev. Lett. 92, 130403 (2004).

${ }^{3}$ I. B. Spielman, W. D. Phillips, and J. V. Porto, Phys. Rev. Lett. 98, 080404 (2007); 100, 120402 (2008).

${ }^{4}$ I. Bloch, J. Dalibard, and W. Zwerger, Rev. Mod. Phys. 80, 885 (2008).

${ }^{5}$ M. P. A. Fisher, P. B. Weichman, G. Grinstein, and D. S. Fisher, Phys. Rev. B 40, 546 (1989).

${ }^{6}$ G. G. Batrouni, R. T. Scalettar, and G. T. Zimanyi, Phys. Rev. Lett. 65, 1765 (1990).

${ }^{7}$ G. G. Batrouni and R. T. Scalettar, Phys. Rev. B 46, 9051 (1992).

${ }^{8}$ J. K. Freericks and H. Monien, Europhys. Lett. 26, 545 (1994).

${ }^{9}$ J. K. Freericks and H. Monien, Phys. Rev. B 53, 2691 (1996).

${ }^{10}$ T. D. Kühner and H. Monien, Phys. Rev. B 58, R14741 (1998).

${ }^{11}$ B. Capogrosso-Sansone, N. V. Prokof'ev, and B. V. Svistunov, Phys. Rev. B 75, 134302 (2007).

${ }^{12}$ B. Capogrosso-Sansone, S. G. Söyler, N. Prokof'ev, and B. Svistunov, Phys. Rev. A 77, 015602 (2008).

${ }^{13}$ G. G. Batrouni, V. Rousseau, R. T. Scalettar, M. Rigol, A. Muramatsu, P. J. H. Denteneer, and M. Troyer, Phys. Rev. Lett. 89, 117203 (2002).

${ }^{14}$ S. Wessel, F. Alet, M. Troyer, and G. G. Batrouni, Phys. Rev. A 70, 053615 (2004).

${ }^{15}$ M. Rigol, G. G. Batrouni, V. G. Rousseau, and R. T. Scalettar,
Phys. Rev. A 79, 053605 (2009).

${ }^{16}$ T.-L. Ho and Q. Zhou, Phys. Rev. Lett. 99, 120404 (2007).

${ }^{17}$ F. Gerbier, Phys. Rev. Lett. 99, 120405 (2007).

${ }^{18}$ M. Aizenman, E. H. Lieb, R. Seiringer, J. P. Solovej, and J. Yngvason, Phys. Rev. A 70, 023612 (2004).

${ }^{19}$ I. Hen and M. Rigol, Phys. Rev. B 80, 134508 (2009).

${ }^{20}$ V. G. Rousseau, D. P. Arovas, M. Rigol, F. Hébert, G. G. Batrouni, and R. T. Scalettar, Phys. Rev. B 73, 174516 (2006).

${ }^{21}$ M. Rigol and A. Muramatsu, Phys. Rev. A 70, 031603(R) (2004); 72, 013604 (2005).

${ }^{22}$ M. Rigol, A. Muramatsu, and M. Olshanii, Phys. Rev. A 74, 053616 (2006).

${ }^{23}$ A. W. Sandvik, Phys. Rev. B 59, R14157 (1999).

${ }^{24}$ A. Dorneich and M. Troyer, Phys. Rev. E 64, 066701 (2001).

${ }^{25}$ T. Matsubara and H. Matsuda, Prog. Theor. Phys. 16, 569 (1956).

${ }^{26}$ E. Lieb, T. Shultz, and D. Mattis, Ann. Phys. (N.Y.) 16, 407 (1961).

${ }^{27}$ D. van Oosten, P. van der Straten, and H. T. C. Stoof, Phys. Rev. A 63, 053601 (2001).

${ }^{28}$ J. K. Freericks, H. R. Krishnamurthy, Y. Kato, N. Kawashima, and N. Trivedi, Phys. Rev. A 79, 053631 (2009).

${ }^{29}$ M. Iskin and J. K. Freericks, Phys. Rev. A 79, 053634 (2009).

${ }^{30}$ S. Fölling, A. Widera, T. Müller, F. Gerbier, and I. Bloch, Phys. Rev. Lett. 97, 060403 (2006).

${ }^{31}$ S. Haley and P. Erdös, Phys. Rev. B 5, 1106 (1972).

${ }^{32}$ K. Sengupta and N. Dupuis, Phys. Rev. A 71, 033629 (2005).

${ }^{33}$ C. Menotti and N. Trivedi, Phys. Rev. B 77, 235120 (2008).

${ }^{34}$ M. Iskin and J. K. Freericks, Phys. Rev. A 80, 063610 (2009). 